\title{
Actualización y mejora de los mecanismos cinéticos de la oxidación de varios compuestos oxigenados
}

\author{
Eduardo Royo, Ángela Millera, Rafael Bilbao, María U. Alzueta \\ Grupo de Procesos Termoquímicos (GPT) \\ Instituto de Investigación en Ingeniería de Aragón (I3A) \\ Universidad de Zaragoza, Mariano Esquillor s/n, 50018, Zaragoza, Spain. \\ Tel. +34-976762707, e-mail: eduardor@unizar.es
}

\begin{abstract}
El Grupo de Procesos Termoquímicos (GPT) lleva años desarrollando mecanismos cinéticos capaces de predecir la conversión de diferentes compuestos orgánicos de interés en procesos de combustión. Modificar el mecanismo cinético de partida puede producir cambios en los procesos de oxidación de especies ya consideradas. En este trabajo, se plantea reconciliar los diferentes mecanismos desarrollados hasta la fecha para obtener un único mecanismo capaz de simular satisfactoriamente la conversión de todas las especies consideradas hasta la actualidad.
\end{abstract}

\section{Introducción}

Un mecanismo cinético-químico de reacción es un conjunto de especies y reacciones químicas que tiene como misión predecir el comportamiento de los reactivos y productos de un sistema de reacción bajo unas condiciones determinadas. El GPT trabaja en el desarrollo de mecanismos cinéticos capaces de predecir la conversión de diversos hidrocarburos y compuestos oxigenados, estos últimos de interés debido a su capacidad para reducir las emisiones contaminantes cuando se usan como aditivos de combustibles de automoción.

El desarrollo de un mecanismo para describir la conversión de un nuevo compuesto implica la introducción de nuevas reacciones y especies al mecanismo, pudiendo producir desajustes a la hora de describir los procesos de oxidación de los compuestos considerados anteriormente. Debido a esto, se considera interesante el asegurar que el mecanismo que se va desarrollando progresivamente siga siendo capaz de simular la conversión de las especies que ya habían sido validadas antes de las modificaciones.

En este contexto, el mecanismo en el que está trabajando actualmente el GPT parte del modelo desarrollado por Glarborg y cols. [1] sobre la reactividad de hidrocarburos con uno o dos átomos de carbono. A dicho mecanismo se le han añadido diferentes subconjuntos de reacciones para poder simular el proceso de oxidación de compuestos tales como: dimetiléter [2], formaldehído [3], metanol [4], etanol [5] y mezclas de acetileno-etanol [6] entre otros. Todos estos subconjuntos de reacciones han sido tomados de los correspondientes mecanismos individuales desarrollados en el GPT para cada una de las especies individuales. Dicho mecanismo ha sido recientemente optimizado para predecir el proceso de oxidación de algunos compuestos oxigenados para condiciones de alta presión [7]. La simulación se ha realizado con el software de cinética química Chemkin Pro.

Tomando este último mecanismo [7], se ha simulado la conversión de varios de los compuestos recogidos en la bibliografía [2-6] y se han detectado discrepancias entre las simulaciones de la conversión de cada compuesto individual recogidas en bibliografía [2-6], y las realizadas con el último mecanismo disponible [7], así como con los resultados experimentales [2-6].

Por ello, es necesario encontrar las causas de las discrepancias que tienen su origen en la progresiva ampliación del mecanismo, mejorar el mecanismo globalmente con objeto de poder predecir de forma adecuada todos los compuestos considerados. Esto permitirá disponer de un único mecanismo adecuado para la simulación de la conversión de un gran número de especies, en lugar de necesitarse mecanismos individuales desarrollados ad-hoc para cada especie. Además, de este modo se dotará de una mayor versatilidad al mecanismo.

\section{Resultados y conclusiones}

Actualmente, la investigación se está centrando en el estudio relativo a dimetiléter y etanol como compuestos principales, ya que los resultados experimentales y simulados en bibliografía [2,5] y los simulados con el actual mecanismo del GPT [7] 
muestran diferencias para condiciones de exceso de aire y mezclas del reactivo correspondiente con NO.

La Figura 1 muestra, a modo de ejemplo, la conversión de dimetiléter y NO para unas condiciones dadas en función de la temperatura. En cada gráfica se representan los resultados experimentales (puntos) [2], los resultados de la simulación con el mecanismo original desarrollado para la conversión de DME (línea discontinua) [2] y la simulación con el mecanismo actual (línea continua) [7].

Considerando la bibliografía disponible, se ha detectado la desaparición de algunas reacciones en el mecanismo actual, las cuales explicaban el comportamiento de la oxidación en los mecanismos antiguos. Tomando como punto de partida estas reacciones se están realizando pruebas, sin ningún resultado determinante por el momento.

\section{REFERENCIAS}

[1]. GLARBORG, P., ALZUETA, M.U., DAMJOHANSEN, K., and MILLER J.A. Kinetic modeling of hydrocarbon/nitric oxide in a flow reactor. Combustion and Flame. 1998, 115(1-2), 1-27.
[2]. ALZUETA, M.U., MURO, J., BILBAO, R., and GLARBORG, P. Oxidation of dimethylether and its interaction with nitrogen oxides. Israel Journal of Chemistry. 1999, 39(1), 73-86.

[3]. GLARBORG, P., ALZUETA, M.U., KJÆRGAARD, K., and DAM-JOHANSEN, K. Oxidation of formaldehyde and its interaction with nitric oxide in a flow reactor. Combustion and Flame. 2002, 132(4), 629-638.

[4]. ALZUETA, M.U., BILBAO, R., and FINESTRA, M. Methanol oxidation and its interaction with nitric oxide. Energy and Fuels. 2001, 15(3), 724-729.

[5]. ALZUETA, M.U., and HERNÁNDEZ, J.M. Ethanol oxidation and its interaction with nitric oxide. Energy and Fuels. 2002, 16(1), 166-171.

[6]. ABIÁN, M., ALZUETA, M.U., MILLERA, Á., BILBAO, R., and ESARTE, C. Oxidation of acetylene-ethanol mixtures and their interaction with NO. Energy and Fuels. 2008, 22(6), 3814-3823.

[7]. MARRODÁN, L., ROYO, E., ALZUETA, M.U., MILLERA, Á., and BILBAO, R. High Pressure oxidation of Dimethoxymethane. Energy and Fuels [en línea]. April 22, 2015 [consulta: 6 mayo 2015]. Disponible en: doi:10.1021/acs.energyfuels.5b00459.

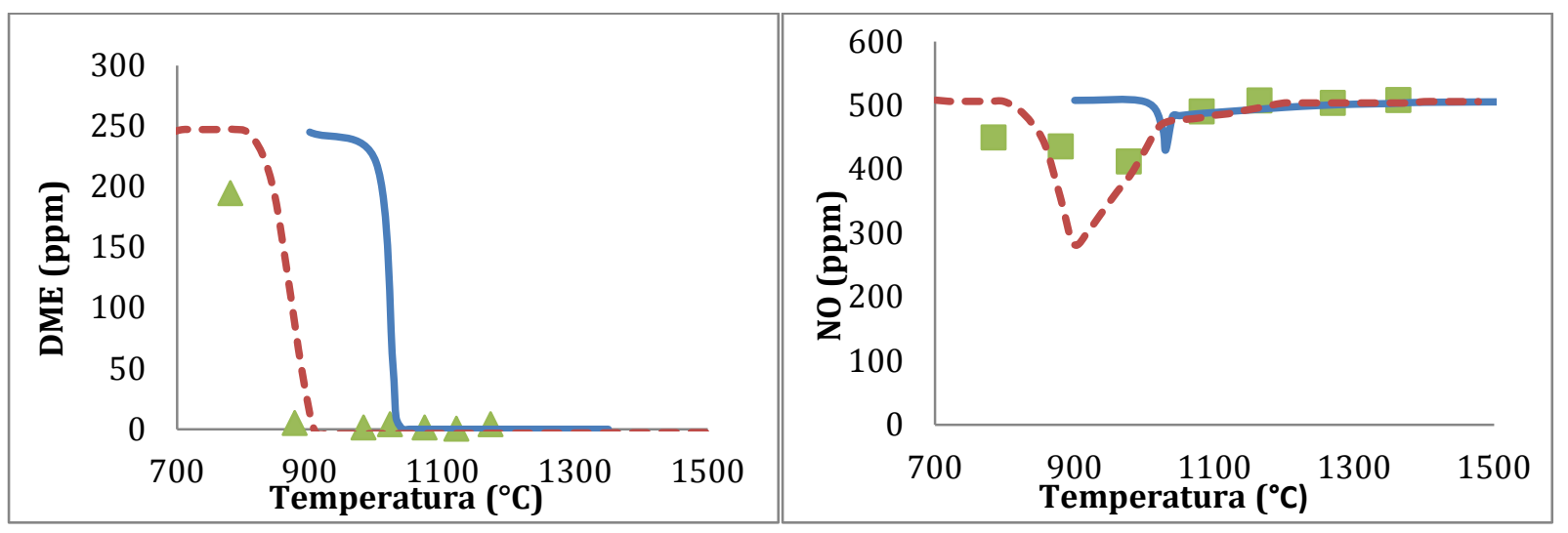

Figura 1. Concentración de DME y NO para el experimento con dimetiléter en condiciones de exceso de aire y mezcla con NO (DME: 245 ppm, $\mathrm{O}_{2}$ : 39400 ppm, NO: 508 ppm). Datos experimentales (puntos), simulación del mecanismo antiguo [2] (línea discontinua) y simulación del mecanismo actual [7] (línea continua). 